\title{
PICARD CONSTANTS OF THREE-SHEETED ALGEBROID SURFACES WITH $p(y)=5$
}

\author{
KAZUNARI SAWADA
}

\begin{abstract}
In 1995 Sawada-Tohge proved that every three-sheeted algebrold Riemann surface with $p(y)=5$ is of Picard constant 5, unless its discrimınant has a form $e^{\delta H}\left(A e^{4 H}+B\right)$, where $\delta=0$ or 1 . In this paper we shall prove that the result remains valid with no condition.
\end{abstract}

\section{Introduction}

Let $\mathfrak{M}(\boldsymbol{R})$ be the family of non-constant meromorphic functions on a Riemann surface $\boldsymbol{R}$. Let $p(f)$ be the cardinal number of values which are not taken by $f \in \mathfrak{M}(\boldsymbol{R})$. Then we put

$$
P(\boldsymbol{R})=\sup _{f \in \mathfrak{M}(\boldsymbol{R})} p(f),
$$

which is called the Picard constant of $\boldsymbol{R}$. We can prove that $P(\boldsymbol{R}) \geqq 2$ if $\boldsymbol{R}$ is open and $P(\boldsymbol{R})=0$ if $\boldsymbol{R}$ is compact. Picard constant plays a very important role in the theory of analytic mappings of Riemann surfaces. Indeed Ozawa [5] proved that there exists no non-trivial analytic mapping of $\boldsymbol{R}$ into $\boldsymbol{S}$ if $P(\boldsymbol{R})<P(\boldsymbol{S})$.

An $n$-sheeted algebroid surface is the proper existence domain of an $n$-valued algebroid function, which is defined by the following irreducible equation;

$$
S_{0}(z) y^{n}-S_{1}(z) y^{n-1}+\cdots+(-1)^{n-1} S_{n-1}(z) y+(-1)^{n} S_{n}(z)=0,
$$

where $S_{i}(z)(i=0,1, \ldots, n)$ are entire functions on $\mathbf{C}$ with no common zeros. An algebroid function $f$ is called transcendental if at least one of $S_{i}(z) / S_{0}(z)$ $(i=1,2, \ldots, n)$ is transcendental and $f$ is called entire if all the $S_{i}(z) / S_{0}(z)$ $(i=1,2, \ldots, n)$ are entire. If $\boldsymbol{R}$ is an $n$-sheeted algebroid surface, then $P(\boldsymbol{R}) \leqq$ $2 n$ by Selberg's theory of algebroid functions [10]. However it is very difficult in general to calculate $P(\boldsymbol{R})$ of a given open Riemann surface $\boldsymbol{R}$, even an algebroid surface.

An $n$-sheeted algebroid surface is called regularly branched if all its branch points are of order $n-1$. Then we have

Recelved May 13, 1999; revised September 14, 1999. 
Theorem A (Aogai [1], Ozawa [6] and Hiromi-Niino [3]). ${ }^{1}$ Let $\boldsymbol{R}$ be an $n$ sheeted regularly branched algebroid surface. If $P(\boldsymbol{R})>3 n / 2$, then $P(\boldsymbol{R})=2 n$ and $\boldsymbol{R}$ can be defined by an algebroid function $y$ such that

$$
y^{n}=\left(e^{H(z)}-\alpha\right)\left(e^{H(z)}-\beta\right)^{n-1}, \quad H(0)=0, \quad \alpha \beta(\alpha-\beta) \neq 0,
$$

where $H(z)$ is a non-constant entire function and $\alpha$ and $\beta$ are constants.

We can prove that there exists no three-sheeted regularly branched surface with $P(\boldsymbol{R})=5$ by theorem $\mathrm{A}$.

In this paper we shall consider three-sheeted algebroid surfaces defined by three-valued entire algebroid functions. Let $\boldsymbol{R}$ be a three-sheeted algebroid Riemann surface defined by

$$
y^{3}-S_{1}(z) y^{2}+S_{2}(z) y-S_{3}(z)=0,
$$

and $\boldsymbol{X}$ be a three-sheeted algebroid Riemann surface defined by

$$
f^{3}-U_{1}(z) f^{2}+U_{2}(z) f-U_{3}(z)=0,
$$

where $S_{i}(z)(i=1,2,3)$ and $U_{j}(z)(j=1,2,3)$ are entire functions. Ozawa and the author proved the following

Theorem B (Ozawa-Sawada [7]). Let $\boldsymbol{X}$ be a three-sheeted algebroid Riemann surface defined by (2). If $p(f)=6$, then we have

$$
\left\{\begin{array}{l}
U_{1}(z)=x_{0} e^{L(z)}+x_{1} \\
U_{2}(z)=b_{1} x_{0} e^{L(z)}+x_{2} \\
U_{3}(z)=x_{3}
\end{array}\right.
$$

where $b_{1}(\neq 0), x_{0}(\neq 0), x_{1}, x_{2}, x_{3}(\neq 0)$ are constants and $L(z)$ is an enture function with $L(0)=0$. And its discriminant $D_{\boldsymbol{X}}$ is

$$
D_{\boldsymbol{X}}=-b_{1}^{2} x_{0}^{4} e^{4 L}+\eta_{3} x_{0}^{3} e^{3 L}+\eta_{2} x_{0}^{2} e^{2 L}+\eta_{1} x_{0} e^{L}+\eta_{0}
$$

where

$$
\left\{\begin{array}{l}
\eta_{3}=4 b_{1}^{3}-2 b_{1}^{2} x_{1}-2 b_{1} x_{2}+4 x_{3} \\
\eta_{2}=12 x_{1} x_{3}-18 b_{1} x_{3}-x_{2}^{2}-4 b_{1} x_{1} x_{2}+12 b_{1}^{2} x_{2}-b_{1}^{2} x_{1}^{2} \\
\eta_{1}=12 x_{1}^{2} x_{3}-18 b_{1} x_{1} x_{3}-18 x_{2} x_{3}-2 x_{1} x_{2}^{2}+12 b_{1} x_{2}^{2}-2 b_{1} x_{1}^{2} x_{2} \\
\eta_{0}=4 x_{1}^{3} x_{3}-x_{1}^{2} x_{2}^{2}+27 x_{3}^{2}-18 x_{1} x_{2} x_{3}+4 x_{2}^{3}(\neq 0)
\end{array}\right.
$$

And we have

\footnotetext{
${ }^{1}$ Ozawa [6] and Hiromi-Niino [3] proved above result in the case $n=2$ and $n=3$ respectively.
} 
Theorem C (Ozawa-Sawada [7]). Let $\boldsymbol{R}$ be a three-sheeted algebroid surface defined by (1). If $p(y)=5$, then we have

$$
\left\{\begin{array}{l}
S_{1}(z)=y_{1} \\
S_{2}(z)=y_{0} e^{H(z)}+y_{2} \\
S_{3}(z)=y_{3}
\end{array}\right.
$$

where $y_{0}(\neq 0), y_{1}, y_{2}, y_{3}(\neq 0)$ are constants and $H(z)$ is a non-constant entire function with $H(0)=0$. We denote this surface by $\boldsymbol{R}_{A}$. Furthermore its discriminant $D_{\boldsymbol{R}_{A}}$ is

$$
D_{\boldsymbol{R}_{A}}=4 y_{0}^{3} e^{3 H}+\zeta_{2} y_{0}^{2} e^{2 H}+\zeta_{1} y_{0} e^{H}+\zeta_{0},
$$

where

$$
\left\{\begin{array}{l}
\zeta_{0}=4 y_{1}^{3} y_{3}-y_{1}^{2} y_{2}^{2}-18 y_{1} y_{2} y_{3}+4 y_{2}^{3}+27 y_{3}^{2}(\neq 0) \\
\zeta_{1}=12 y_{2}^{2}-18 y_{1} y_{3}-2 y_{1}^{2} y_{2} \\
\zeta_{2}=12 y_{2}-y_{1}^{2}
\end{array}\right.
$$

Remark. Ozawa-Sawada [7] proved that there exist the following three surfaces $\boldsymbol{R}_{A}, \boldsymbol{R}_{B}$ and $\boldsymbol{R}_{G}$ with $p(y)=5$ :

$$
\begin{gathered}
\boldsymbol{R}_{A}: y^{3}-y_{1} y^{2}+\left(y_{0} e^{H(z)}+y_{2}\right) y-y_{3}=0, \\
\boldsymbol{R}_{B}: y^{3}-\left(z_{0} e^{H(z)}+z_{1}\right) y^{2}+z_{2} y-z_{3}=0,
\end{gathered}
$$

and

$$
\boldsymbol{R}_{G}: y^{3}-\left(w_{0} e^{-H(z)}+a\right) y^{2}+w_{1} w_{0} e^{-H(z)} y-w_{2} w_{0} e^{-H(z)}=0,
$$

where $H(z)$ is a non-constant entire function and $y_{0}(\neq 0), y_{1}, y_{2}, y_{3}(\neq 0)$, $z_{0}(\neq 0), z_{1}, z_{2}, z_{3}(\neq 0), \quad a(\neq 0), w_{0}(\neq 0), w_{1}$ and $w_{2}(\neq 0)$ are constants. Furthermore

$$
x^{3}-y_{1} x^{2}+y_{2} x-y_{3}=0
$$

has 3 distinct solutions.

However we may consider 'only one' surface $\boldsymbol{R}_{A}$. In fact we can investigate that $\boldsymbol{R}_{A}, \boldsymbol{R}_{B}$ and $\boldsymbol{R}_{G}$ are conformally equivalent. Putting $y=1 / Y$, then we can deduce $\boldsymbol{R}_{B}$ from $\boldsymbol{R}_{A}$. And putting $y=A(1-a / Y)$, where $A$ is a solution of $A^{3}-y_{1} A^{2}+y_{2} A-y_{3}=0$, then we can deduce $\boldsymbol{R}_{G}$ from $\boldsymbol{R}_{A}$.

Furthermore we have

THEOREM D (Ozawa-Sawada [7], Sawada-Tohge [9]). ${ }^{2}$ Let $\boldsymbol{R}$ be the surface defined by (1) with $p(y)=5$. If $\left(\zeta_{1}, \zeta_{2}\right) \neq(0,0)$, then $P(\boldsymbol{R})=5$.

\footnotetext{
${ }^{2}$ Ozawa-Sawada [7] proved the above result under the condition that $\boldsymbol{R}$ is of finite order and Sawada-Tohge [9] proved that the result remains valid without the order condition.
} 
In this paper we shall prove that the above result remains valid without the condition $\left(\zeta_{1}, \zeta_{2}\right) \neq(0,0)$. In fact we shall prove the following

THEOREM. The surface $\boldsymbol{R}_{A}$ is of Picard constant 5 with no condition.

\section{Preparations}

In this paper we shall consider the surfaces, defined by theorem $\mathrm{C}$, satisfying the additional condition $\zeta_{1}=\zeta_{2}=0$ and the surfaces, defined by theorem B, satisfying the additional condition $\eta_{1}=\eta_{2}=\eta_{3}=0$. First of all we list up all the surfaces $X$ defined by (2) and (3) with the condition $\eta_{1}=\eta_{2}=\eta_{3}=0$. By (4) we have

$$
\left\{\begin{array}{l}
\eta_{3}=4 b_{1}^{3}-2 b_{1}^{2} x_{1}-2 b_{1} x_{2}+4 x_{3}=0 \\
\eta_{2}=12 x_{1} x_{3}-18 b_{1} x_{3}-x_{2}^{2}-4 b_{1} x_{1} x_{2}+12 b_{1}^{2} x_{2}-b_{1}^{2} x_{1}^{2}=0 \\
\eta_{1}=12 x_{1}^{2} x_{3}-18 b_{1} x_{1} x_{3}-18 x_{2} x_{3}-2 x_{1} x_{2}^{2}+12 b_{1} x_{2}^{2}-2 b_{1} x_{1}^{2} x_{2}=0
\end{array}\right.
$$

To eliminate $x_{3}$ from $\eta_{1}=0$ and $\eta_{3}=0$, let us calculate the resultant of $\eta_{1}=0$ and $\eta_{3}=0$, then we have

$$
\left(3 b_{1}-2 x_{1}\right)\left(6 b_{1}^{2}-3 b_{1} x_{1}+x_{2}\right)\left(b_{1} x_{1}+x_{2}\right)=0 .
$$

Similarly eliminating $x_{3}$ from $\eta_{2}=0$ and $\eta_{3}=0$, we have

$$
18 b_{1}^{4}-21 b_{1}^{3} x_{1}+5 b_{1}^{2} x_{1}^{2}+3 b_{1}^{2} x_{2}+2 b_{1} x_{1} x_{2}-x_{2}^{2}=0 .
$$

First of all we assume that $3 b_{1}-2 x_{1}=0$. Let us put $B=b_{1}$, then $x_{1}=3 B / 2$. And from $x_{1}=3 B / 2$ and (7), we have $x_{2}=d B^{2}$, where $d$ is a constant such that $4 d^{2}-24 d+9=0$. Furthermore we have $x_{3}=(2 d-1) B^{3} / 4$ from $\eta_{3}=0$.

Next we assume that $6 b_{1}^{2}-3 b_{1} x_{1}+x_{2}=0$. Eliminating $x_{2}$ from $6 b_{1}^{2}-$ $3 b_{1} x_{1}+x_{2}=0$ and (7), we have

$$
b_{1}^{2}\left(18 b_{1}^{2}-6 b_{1} x_{1}-x_{1}^{2}\right)=0 .
$$

Similarly we put $B=b_{1}$, then $x_{1}=d B$, where $d$ is a constant such that $d^{2}+$ $6 d-18=0$. Furthermore we have $x_{2}=3(d-2) B^{2}$ and $x_{3}=2(d-2) B^{3}$ from $6 b_{1}^{2}-3 b_{1} x_{1}+x_{2}=0$ and $\eta_{3}=0$, respectively.

Last we assume that $b_{1} x_{1}+x_{2}=0$. Eliminating $x_{2}$ from $b_{1} x_{1}+x_{2}=0$ and (7), we have

$$
b_{1}^{2}\left(9 b_{1}^{2}-12 b_{1} x_{1}+x_{1}^{2}\right)=0 .
$$

Therefore, putting $B=b_{1}$, we have $x_{1}=d B$ and $x_{2}=-d B^{2}$, where $d$ is a constant such that $d^{2}-12 d+9=0$. Furthermore we have $x_{3}=-B^{3}$ from $\eta_{3}=0$. Therefore there exist only three surfaces $\boldsymbol{X}$ satisfying the condition $\eta_{1}=\eta_{2}=\eta_{3}=0$ : 


$$
\boldsymbol{X} \text {-(i) }\left\{\begin{array}{l}
U_{1}(z)=x_{0} e^{L(z)}+\frac{3}{2} B, \\
U_{2}(z)=B x_{0} e^{L(z)}+d B^{2}, \\
U_{3}(z)=\frac{2 d-1}{4} B^{3},
\end{array}\right.
$$

where $B(\neq 0)$ is a constant and $d$ is a solution of $4 d^{2}-24 d+9=0$, and its discriminant is

$$
\begin{gathered}
D_{X-(\mathrm{i})}=-B^{2} x_{0}^{4} e^{4 L}+\frac{1}{16}(4 d-3)^{3} B^{6}, \\
X \text {-(ii) }\left\{\begin{array}{l}
U_{1}(z)=x_{0} e^{L(z)}+d B, \\
U_{2}(z)=B x_{0} e^{L(z)}+3(d-2) B^{2}, \\
U_{3}(z)=2(d-2) B^{3},
\end{array}\right.
\end{gathered}
$$

where $B(\neq 0)$ is a constant and $d$ is a solution of $d^{2}+6 d-18=0$, and its discriminant is

$$
D_{X \text {-(ii) }}=-B^{2} x_{0}^{4} e^{4 L}-(d-2)(d-6)^{3} B^{6},
$$

and

$$
\boldsymbol{X} \text {-(iii) }\left\{\begin{array}{l}
U_{1}(z)=x_{0} e^{L(z)}+d B \\
U_{2}(z)=B x_{0} e^{L(z)}-d B^{2} \\
U_{3}(z)=-B^{3}
\end{array}\right.
$$

where $B(\neq 0)$ is a constant and $d$ is a solution of $d^{2}-12 d+9=0$, and its discriminant is

$$
D_{X \text {-(iii) }}=-B^{2} x_{0}^{4} e^{4 L}-(d-1)(d+3)^{3} B^{6} .
$$

Next we list up all the surfaces $\boldsymbol{R}_{A}$ defined by (1) and (5) with the condition $\zeta_{1}=\zeta_{2}=0$. By $(6)$ we have

$$
\left\{\begin{array}{l}
\zeta_{1}=12 y_{2}^{2}-18 y_{1} y_{3}-2 y_{1}^{2} y_{2}=0 \\
\zeta_{2}=12 y_{2}-y_{1}^{2}=0 .
\end{array}\right.
$$

If $y_{1}=0$, we have $y_{2}=0$ and $y_{3}=A$, where $A$ is a non-zero constant. If $y_{1} \neq 0$, putting $y_{1}=6 A(\neq 0)$, we have $y_{2}=3 A^{2}$ from $\zeta_{2}=0$ and $y_{3}=-A^{3}$ from $\zeta_{1}=0$. Therefore there exist only two surfaces $\boldsymbol{R}_{A}$ satisfying the condition $\zeta_{1}=\zeta_{2}=0$ : 


$$
\boldsymbol{R}_{A^{-}}(\mathrm{i})\left\{\begin{array}{l}
S_{1}(z)=0 \\
S_{2}(z)=y_{0} e^{H(z)}, \\
S_{3}(z)=A
\end{array}\right.
$$

where $A(\neq 0)$ is a constant. Its discriminant is

$$
D_{\boldsymbol{R}_{A}-(\mathrm{i})}=4 y_{0}^{3} e^{3 H}+27 A^{2} \text {, }
$$

and

$$
\boldsymbol{R}_{A^{-}} \text {(ii) }\left\{\begin{array}{l}
S_{1}(z)=6 A \\
S_{2}(z)=y_{0} e^{H(z)}+3 A^{2}, \\
S_{3}(z)=-A^{3},
\end{array}\right.
$$

where $A(\neq 0)$ is a constant. Its discriminant is

$$
D_{\boldsymbol{R}_{A} \text {-(ii) }}=4 y_{0}^{3} e^{3 H}-729 A^{6} \text {. }
$$

Now we suppose that $\boldsymbol{R}$, defined by theorem $C$, is of Picard constant 6 . There exists a meromorphic function $f$ on $\boldsymbol{R}$ such that $p(f)=6$. Without loss of generality we may assume that the function $f$ is entire, which does not take 5 finite values. The function $f$ can be represented by

$$
f=f_{0}+f_{1} y+f_{2} y^{2}
$$

where $f_{0}, f_{1}$ and $f_{2}$ are "single-valued" meromorphic functions, which have poles at most on $\left\{z \mid H^{\prime}(z)=0\right\}$ (see Ozawa-Sawada [7]). Eliminating $y$ from (1) and (8), we have

$$
f^{3}-U_{1} f^{2}+U_{2} f-U_{3}=0
$$

where

$$
\begin{aligned}
U_{1}= & 3 f_{0}+f_{1} S_{1}+f_{2}\left(S_{1}^{2}-2 S_{2}\right), \\
U_{2}= & 3 f_{0}^{2}+2 f_{0}\left\{f_{1} S_{1}+f_{2}\left(S_{1}^{2}-2 S_{2}\right)\right\}+f_{1}^{2} S_{2}+f_{1} f_{2}\left(S_{1} S_{2}-3 S_{3}\right) \\
& +f_{2}^{2}\left(S_{2}^{2}-2 S_{1} S_{3}\right) \\
U_{3}= & f_{0}^{3}+f_{0}^{2}\left\{f_{1} S_{1}+f_{2}\left(S_{1}^{2}-2 S_{2}\right)\right\} \\
& +f_{0}\left\{f_{1}^{2} S_{2}+f_{1} f_{2}\left(S_{1} S_{2}-3 S_{3}\right)+f_{2}^{2}\left(S_{2}^{2}-2 S_{1} S_{3}\right)\right\} \\
& +f_{1}^{3} S_{3}+f_{1}^{2} f_{2} S_{1} S_{3}+f_{1} f_{2}^{2} S_{2} S_{3}+f_{2}^{3} S_{3}^{2} .
\end{aligned}
$$

Because of $p(f)=6$, the function $f$ defines the surface $\boldsymbol{X}$ described by theorem B. And we have the following relation between the discriminants of $\boldsymbol{R}$ and $\boldsymbol{X}$ (see Ozawa-Sawada [7]):

$$
D_{X}=D_{R} \cdot G^{2}
$$


where

$$
G=f_{1}^{3}+2 f_{1}^{2} f_{2} S_{1}+\left(S_{1}^{2}+S_{2}\right) f_{1} f_{2}^{2}+\left(S_{1} S_{2}-S_{3}\right) f_{2}^{3} .
$$

Now we may assume that the surface $\boldsymbol{R}$ satisfies the condition $\zeta_{1}=\zeta_{2}=0$, then we have that the surface $\boldsymbol{X}$ satisfies the condition $\eta_{1}=\eta_{2}=\eta_{3}=0$ and $G=$ $K e^{M}$, where $K$ is a non-zero constant and $M$ is an entire function with $M(0)=0$ (see Sawada-Tohge [9]).

Eliminating $f_{0}$ from (9) and (10), we have

$$
\begin{aligned}
& -3 f_{1}^{2}\left(S_{1}^{2}-3 S_{2}\right)-3 f_{1} f_{2}\left(2 S_{1}^{3}-7 S_{1} S_{2}+9 S_{3}\right) \\
& \quad-3 f_{2}^{2}\left(S_{1}^{4}-4 S_{1}^{2} S_{2}+S_{2}^{2}+6 S_{1} S_{3}\right)+3 U_{1}^{2}-9 U_{2}=0 .
\end{aligned}
$$

Similarly eliminating $f_{0}$ from (9) and (11) we have

$$
\begin{aligned}
f_{1}^{3}\left(2 S_{1}^{3}-9 S_{1} S_{2}+27 S_{3}\right) & \\
+ & 3 f_{1}^{2}\left\{2 f_{2}\left(S_{1}^{4}-5 S_{1}^{2} S_{2}+3 S_{2}^{2}+9 S_{1} S_{3}\right)-U_{1}\left(S_{1}^{2}-3 S_{2}\right)\right\} \\
& +3 f_{1} f_{2}\left\{f_{2}\left(2 S_{1}^{5}-11 S_{1}^{3} S_{2}+15 S_{1}^{2} S_{3}+11 S_{1} S_{2}^{2}-9 S_{2} S_{3}\right)\right. \\
& \left.\quad-U_{1}\left(2 S_{1}^{3}-7 S_{1} S_{2}+9 S_{3}\right)\right\} \\
& +f_{2}^{3}\left(2 S_{1}^{6}-12 S_{1}^{4} S_{2}+18 S_{1}^{3} S_{3}+15 S_{1}^{2} S_{2}^{2}-36 S_{1} S_{2} S_{3}+2 S_{2}^{3}+27 S_{3}^{2}\right) \\
& -3 f_{2}^{2} U_{1}\left(S_{1}^{4}-4 S_{1}^{2} S_{2}+S_{2}^{2}+S_{1} S_{3}\right)+U_{1}^{3}-27 U_{3}=0 .
\end{aligned}
$$

We can construct the following liner equation with respect to $f_{1}$ from (13) and (14):

$$
\begin{aligned}
\frac{1}{\left(S_{1}^{2}-3 S_{2}\right)^{2}}[ & f_{1}\left\{-3 f_{2}^{2}\left(4 S_{1}^{3} S_{3}-S_{1}^{2} S_{2}^{2}-18 S_{1} S_{2} S_{3}+4 S_{2}^{3}+27 S_{3}^{2}\right)\right. \\
& \left.\quad-\left(S_{1}^{2}-3 S_{2}\right)\left(U_{1}^{2}-3 U_{2}\right)\right\} \\
& -2 f_{2}^{3} S_{1}\left(4 S_{1}^{3} S_{3}-S_{1}^{2} S_{2}^{2}-18 S_{1} S_{2} S_{3}+4 S_{2}^{3}+27 S_{3}^{2}\right) \\
& \left.-f_{2}\left(S_{1} S_{2}-9 S_{3}\right)\left(U_{1}^{2}-3 U_{2}\right)+G\left(S_{1}^{2}-3 S_{2}\right)^{2}\right]=0 .
\end{aligned}
$$

Similarly we can construct the following linear equation with respect to $f_{1}$ from (13) and (15):

$$
\begin{aligned}
& \left.\frac{1}{\left(S_{1}^{2}-\right.}-3 S_{2}\right)^{2}\left\{2 f_{2}\left(S_{1}^{2}-3 S_{2}\right)-3 U_{1}\right\} \\
& \times\left[f _ { 1 } \left\{4 f_{2}^{3}\left(S_{1}^{2}-3 S_{2}\right)\left(4 S_{1}^{3} S_{3}-S_{1}^{2} S_{2}^{2}-18 S_{1} S_{2} S_{3}+4 S_{2}^{3}+27 S_{3}^{2}\right)\right.\right. \\
& \quad-9 f_{2}^{2} U_{1}\left(4 S_{1}^{3} S_{3}-S_{1}^{2} S_{2}^{2}-18 S_{1} S_{2} S_{3}+4 S_{2}^{3}+27 S_{3}^{2}\right) \\
& \left.\quad-\left(S_{1}^{2}-3 S_{2}\right)\left(U_{1}^{3}-27 U_{3}\right)-G\left(S_{1}^{2}-3 S_{2}\right)\left(2 S_{1}^{3}-9 S_{1} S_{2}+27 S_{3}\right)\right\} \\
& +2 f_{2}^{4}\left(2 S_{1}^{3}-7 S_{1} S_{2}+9 S_{3}\right)\left(4 S_{1}^{3} S_{3}-S_{1}^{2} S_{2}^{2}-18 S_{1} S_{2} S_{3}+4 S_{2}^{3}+27 S_{3}^{2}\right)
\end{aligned}
$$




$$
\begin{aligned}
& -6 f_{2}^{3} S_{1} U_{1}\left(4 S_{1}^{3} S_{3}-S_{1}^{2} S_{2}^{2}-18 S_{1} S_{2} S_{3}+4 S_{2}^{3}+27 S_{3}^{2}\right) \\
& +f_{2}\left\{G \left(-2 S_{1}^{6}+16 S_{1}^{4} S_{2}+18 S_{1}^{3} S_{3}-45 S_{1}^{2} S_{2}^{2}-108 S_{1} S_{2} S_{3}\right.\right. \\
& \left.\left.\quad+54 S_{2}^{3}+243 S_{3}^{2}\right)-\left(S_{1} S_{2}-9 S_{3}\right)\left(U_{1}^{3}-27 U_{3}\right)\right\} \\
& \left.+3 G U_{1}\left(S_{1}^{2}-3 S_{2}\right)^{2}\right]=0 .
\end{aligned}
$$

Therefore eliminating $f_{1}$ from (16) and (17) we have the following equation, which plays an important role:

$$
\frac{E_{1} \cdot E_{2}}{\left(S_{1}^{2}-3 S_{2}\right)^{4}\left\{2 f_{2}\left(S_{1}^{2}-3 S_{2}\right)-3 U_{1}\right\}}=0,
$$

where

$$
\begin{aligned}
E_{1}= & f_{2}^{3}\left(4 S_{1}^{3} S_{3}-S_{1}^{2} S_{2}^{2}-18 S_{1} S_{2} S_{3}+4 S_{2}^{3}+27 S_{3}^{2}\right) \\
& \times\left(2 S_{1}^{3}-9 S_{1} S_{2}+27 S_{3}\right)-G\left(S_{1}^{2}-3 S_{2}\right)^{3}
\end{aligned}
$$

and

$$
\begin{aligned}
E_{2}= & 2\left(4 S_{1}^{3} S_{3}-S_{1}^{2} S_{2}^{2}-18 S_{1} S_{2} S_{3}+4 S_{2}^{3}+27 S_{3}^{2}\right) f_{2}^{3} \\
& +2\left(U_{1}^{2}-3 U_{2}\right)\left(S_{1}^{2}-3 S_{2}\right) f_{2} \\
& +\left(2 S_{1}^{3}-9 S_{1} S_{2}+27 S_{3}\right) G-\left(2 U_{1}^{3}-9 U_{1} U_{2}+27 U_{3}\right) .
\end{aligned}
$$

It is easy to verify that there exists no single-valued meromorphic function $f_{2}$ satisfying $E_{1}=0$. In fact, in the case $\boldsymbol{R}=\boldsymbol{R}_{A^{-}}$(i), we have

$$
27 A f_{2}^{3}\left(4 y_{0}^{3} e^{3 H}+27 A^{2}\right)+27 G y_{0}^{3} e^{3 H}=0
$$

from (19). In this case the function $f_{2}$ mast have an algebraic branch point of order 2 at every zero of $4 y_{0}^{3} e^{3 H}+27 A^{2}$, because that the function $G=K e^{M}$ has no zero. This is a contradiction. And, in the case $\boldsymbol{R}=\boldsymbol{R}_{A^{-}}$-(ii), we have

$$
27 A f_{2}^{3}\left(2 y_{0} e^{H}-9 A^{2}\right)\left(4 y_{0}^{3} e^{3 H}-729 A^{6}\right)-27 G\left(y_{0} e^{H}-9 A^{2}\right)^{3}=0,
$$

from (19). In this case the function $f_{2}$ mast have an algebraic branch point of order 2 at every zero of $4 y_{0}^{3} e^{3 H}-729 A^{6}$ and $2 y_{0} e^{H}-9 A^{2}$. This is also a contradiction.

In the following section we shall consider the equation $E_{2}=0$. And we shall prove that there exists no single-valued meromorphic function $f_{2}$ satisfying the equation $E_{2}=0$.

\section{Proof of theorem}

In this section we shall consider the following equation

$$
\begin{aligned}
E_{2}= & 2\left(4 S_{1}^{3} S_{3}-S_{1}^{2} S_{2}^{2}-18 S_{1} S_{2} S_{3}+4 S_{2}^{3}+27 S_{3}^{2}\right) f_{2}^{3} \\
& +2\left(U_{1}^{2}-3 U_{2}\right)\left(S_{1}^{2}-3 S_{2}\right) f_{2} \\
& +\left(2 S_{1}^{3}-9 S_{1} S_{2}+27 S_{3}\right) G-\left(2 U_{1}^{3}-9 U_{1} U_{2}+27 U_{3}\right)=0
\end{aligned}
$$


where $S_{i}(i=1,2,3)$ are entire functions, the pair of which defines either $\boldsymbol{R}_{A}$-(i) or $\boldsymbol{R}_{A}$-(ii), $U_{j}(j=1,2,3)$ are entire functions, the pair of which defines one of the surfaces $X$-(i), $X$-(ii) and $X$-(iii) and $G=K e^{M}$. We shall prove that there exists no single-valued meromorphic function $f_{2}$ satisfying the equation (20).

Let us consider the case $\boldsymbol{R}=\boldsymbol{R}_{A^{-}}$(i) and $\boldsymbol{X}=\boldsymbol{X}$-(i). Then we have

$$
-B^{2} x_{0}^{4} e^{4 L}+\frac{1}{16}(4 d-3)^{3} B^{6}=\left(4 y_{0}^{3} e^{3 H}+27 A^{2}\right) \cdot K^{2} e^{2 M}
$$

from (12) and their discriminants of $\boldsymbol{R}_{A}$-(i) and $\boldsymbol{X}$-(i). In this case there exist only two possibilities:

$$
\text { (I) }\left\{\begin{array} { l } 
{ M \equiv 0 , } \\
{ 4 L \equiv 3 H , } \\
{ - B ^ { 2 } x _ { 0 } ^ { 4 } = 4 y _ { 0 } ^ { 3 } K ^ { 2 } , } \\
{ \frac { 1 } { 1 6 } ( 4 d - 3 ) ^ { 3 } B ^ { 6 } = 2 7 A ^ { 2 } K ^ { 2 } , }
\end{array} \quad ( \text { II } ) \quad \left\{\begin{array}{l}
2 M \equiv 4 L \equiv-3 H, \\
-B^{2} x_{0}^{4}=27 A^{2} K^{2}, \\
\frac{1}{16}(4 d-3)^{3} B^{6}=4 y_{0}^{3} K^{2} .
\end{array}\right.\right.
$$

First of all we consider the case (I). Let us put $J=L / 3=H / 4, X=e^{J}$ and $w=f_{2}$. Then we have the following algebraic equation:

$$
\begin{gathered}
2\left(4 y_{0}^{3} X^{12}+27 A^{2}\right) w^{3}-\frac{3}{2} y_{0} /\left(4 x_{0}^{2} X^{6}-3(4 d-3) B^{2}\right) X^{4} w \\
-2 x_{0}^{3} X^{9}+9 d B^{2} x_{0} X^{3}+27 A K=0
\end{gathered}
$$

from (20). Next we consider the case (II) and let us put $J=-L / 3=H / 4=$ $-M / 6, X=e^{J}$ and $w=f_{2}$. Then, from (20), we have

$$
\begin{gathered}
2\left(4 y_{0}^{3} X^{12}+27 A^{2}\right) w^{3}+\frac{3}{2} y_{0} \frac{3(4 d-3) B^{2} X^{6}-4 x_{0}^{2}}{X^{2}} w \\
+\frac{9 d B^{2} x_{0} X^{6}+27 A K X^{3}-2 x_{0}^{3}}{X^{9}}=0
\end{gathered}
$$

and

$$
\begin{aligned}
& 2\left(4 y_{0}^{3} X^{12}+27 A^{2}\right) X^{9} w^{3}+\frac{3}{2} y_{0}\left(3(4 d-3) B^{2} X^{6}-4 x_{0}^{2}\right) X^{7} w \\
& +9 d B^{2} x_{0} X^{6}+27 A K X^{3}-2 x_{0}^{3}=0 .
\end{aligned}
$$

In the case $\boldsymbol{R}=\boldsymbol{R}_{A}$-(i) and $\boldsymbol{X}=\boldsymbol{X}$-(ii), by the similar way of above, we have the following two algebraic equations:

$$
\begin{aligned}
& 2\left(4 y_{0}^{3} X^{12}+27 A^{2}\right) w^{3} \\
& \quad-6 y_{0}\left(x_{0}^{2} X^{6}+(2 d-3) B x_{0} X^{3}+(d-3)(d-6) B^{2}\right) X^{4} w \\
& \quad-2 x_{0}^{3} X^{9}-3(2 d-3) B x_{0}^{2} X^{6}-6(d-3)^{2} B^{2} x_{0} X^{3} \\
& \quad-(d-6)^{2}(2 d-3) B^{3}+27 A K=0,
\end{aligned}
$$


where $M \equiv 0, J=L / 3=H / 4, X=e^{J}$ and $w=f_{2}$, and

$$
\begin{aligned}
& 2\left(4 y_{0}^{3} X^{12}+27 A^{2}\right) X^{9} w^{3} \\
& \quad-6 y_{0}\left((d-3)(d-6) B^{2} X^{6}+(2 d-3) B x_{0} X^{3}+x_{0}^{2}\right) X^{7} w \\
& \quad-(d-6)^{2}(2 d-3) B^{3} X^{9}-6(d-3)^{2} B^{2} x_{0} X^{6} \\
& -3\left((2 d-3) B x_{0}^{2}-9 A K\right) X^{3}-2 x_{0}^{3}=0,
\end{aligned}
$$

where $J=-L / 3=H / 4=-M / 6, X=e^{J}$ and $w=f_{2}$, from (20).

Similarly, in the case $\boldsymbol{R}=\boldsymbol{R}_{A}$-(i) and $\boldsymbol{X}=\boldsymbol{X}$-(iii), we have

$$
\begin{aligned}
& 2\left(4 y_{0}^{3} X^{12}+27 A^{2}\right) w^{3} \\
& -6 y_{0}\left(x_{0}^{2} X^{6}+(2 d-3) B x_{0} X^{3}+d(d+3) B^{2}\right) X^{4} w \\
& -2 x_{0}^{3} X^{9}-3(2 d-3) B x_{0}^{2} X^{6}-6 d^{2} B^{2} x_{0} X^{3} \\
& \quad-(d+3)^{2}(2 d-3) B^{3}+27 A K=0,
\end{aligned}
$$

where $M \equiv 0, J=L / 3=H / 4, X=e^{J}$ and $w=f_{2}$, and

$$
\begin{aligned}
2\left(4 y_{0}^{3}\right. & \left.X^{12}+27 A^{2}\right) X^{9} w^{3} \\
& -6 y_{0}\left(d(d+3) B^{2} X^{6}+(2 d-3) B x_{0} X^{3}+x_{0}^{2}\right) X^{7} w \\
& -(d+3)^{2}(2 d-3) B^{3} X^{9}-6 d^{2} B^{2} x_{0} X^{6} \\
& -3\left((2 d-3) B x_{0}^{2}-9 A K\right) X^{3}-2 x_{0}^{3}=0,
\end{aligned}
$$

where $J=-L / 3=H / 4=-M / 6, X=e^{J}$ and $w=f_{2}$.

Similarly, in the case $\boldsymbol{R}=\boldsymbol{R}_{A^{-}}$-(ii) and $\boldsymbol{X}=\boldsymbol{X}$-(i), we have

$$
\begin{gathered}
2\left(4 y_{0}^{3} X^{12}-729 A^{6}\right) w^{3}-\frac{3}{2}\left(y_{0} X^{4}-9 A^{2}\right)\left(4 x_{0}^{2} X^{6}-3(4 d-3) B^{2}\right) w \\
-2 x_{0}^{3} X^{9}-54 A K y_{0} X^{4}+9 d B^{2} x_{0} X^{3}+243 A^{3} K=0,
\end{gathered}
$$

where $M \equiv 0, J=L / 3=H / 4, X=e^{J}$ and $w=f_{2}$, and

$$
\begin{aligned}
2\left(4 y_{0}^{3} X^{12}-729 A^{6}\right) X^{9} w^{3} & \\
+ & \frac{3}{2}\left(y_{0} X^{4}-9 A^{2}\right)\left(3(4 d-3) B^{2} X^{6}-4 x_{0}^{2}\right) X^{3} w \\
& -54 A K y_{0} X^{7}+9 d B^{2} x_{0} X^{6}+243 A^{3} K X^{3}-2 x_{0}^{3}=0
\end{aligned}
$$

where $J=-L / 3=H / 4=-M / 6, X=e^{J}$ and $w=f_{2}$.

Similarly, in the case $\boldsymbol{R}=\boldsymbol{R}_{A}$-(ii) and $\boldsymbol{X}=\boldsymbol{X}$-(ii), we have 


$$
\begin{aligned}
2\left(4 y_{0}^{3}\right. & \left.X^{12}-729 A^{6}\right) w^{3} \\
& -6\left(y_{0} X^{4}-9 A^{2}\right)\left(x_{0}^{2} X^{6}+(2 d-3) B x_{0} X^{3}+(d-3)(d-6) B^{2}\right) w \\
& -2 x_{0}^{3} X^{9}-3(2 d-3) B x_{0}^{2} X^{6}-54 A K y_{0} X^{4}-6(d-3)^{2} B^{2} x_{0} X^{3} \\
& -(d-6)^{2}(2 d-3) B^{3}+243 A^{3} K=0,
\end{aligned}
$$

where $M \equiv 0, J=L / 3=H / 4, X=e^{J}$ and $w=f_{2}$, and

$$
\begin{aligned}
& 2\left(4 y_{0}^{3} X^{12}-729 A^{6}\right) X^{9} w^{3} \\
& \quad-6\left(y_{0} X^{4}-9 A^{2}\right)\left((d-3)(d-6) B^{2} X^{6}+(2 d-3) B x_{0} X^{3}+x_{0}^{2}\right) X^{3} w \\
& \quad-(d-6)^{2}(2 d-3) B^{3} X^{9}-54 A K y_{0} X^{7}-6(d-3)^{2} B^{2} x_{0} X^{6} \\
& \quad-3\left((2 d-3) B x_{0}^{2}-81 A^{3} K\right) X^{3}-2 x_{0}^{3}=0,
\end{aligned}
$$

where $J=-L / 3=H / 4=-M / 6, X=e^{J}$ and $w=f_{2}$.

Similarly, in the case $\boldsymbol{R}=\boldsymbol{R}_{A^{-}}$(ii) and $\boldsymbol{X}=\boldsymbol{X}$-(iii), we have

$$
\begin{aligned}
2\left(4 y_{0}^{3}\right. & \left.X^{12}-729 A^{6}\right) w^{3} \\
& -6\left(y_{0} X^{4}-9 A^{2}\right)\left(x_{0}^{2} X^{6}+(2 d-3) B x_{0} X^{3}+d(d+3) B^{2}\right) w \\
& -2 x_{0}^{3} X^{9}-3(2 d-3) B x_{0}^{2} X^{6}-54 A K y_{0} X^{4}-6 d^{2} B^{2} x_{0} X^{3} \\
& -(d+3)^{2}(2 d-3) B^{3}+243 A^{3} K=0,
\end{aligned}
$$

where $M \equiv 0, J=L / 3=H / 4, X=e^{J}$ and $w=f_{2}$, and

$$
\begin{aligned}
& 2\left(4 y_{0}^{3} X^{12}-729 A^{6}\right) X^{9} w^{3} \\
& \quad-6\left(y_{0} X^{4}-9 A^{2}\right)\left(d(d+3) B^{2} X^{6}+(2 d-3) B x_{0} X^{3}+x_{0}^{2}\right) X^{3} w \\
& \quad-(d+3)^{2}(2 d-3) B^{3} X^{9}-54 A K y_{0} X^{7}-6 d^{2} B^{2} x_{0} X^{6} \\
& \quad-3\left((2 d-3) B x_{0}^{2}-81 A^{3} K\right) X^{3}-2 x_{0}^{3}=0,
\end{aligned}
$$

where $J=-L / 3=H / 4=-M / 6, X=e^{J}$ and $w=f_{2}$.

Now we need the following

LEMma 1 (Picard [8]). If the curve $\varphi(X, w)=0$ is of genus $g>1$, then there exists no pair of meromorphic functions $X(z)$ and $w(z)$ such that $\varphi(X(z), w(z)) \equiv 0$.

Proof of Theorem. Let $\boldsymbol{R}$ be the surface $\boldsymbol{R}_{A}$-(i). And let us assume that $\boldsymbol{R}$ is of Picard constant 6. Then there exists an entire function $f=f_{0}+f_{1} y+f_{2} y^{2}$ on $\boldsymbol{R}$, which does not take 5 finite values. Furthermore we assume that the 
function $f$ defines the surface $\boldsymbol{X}=\boldsymbol{X}$-(i). In this case the single-valued meromorphic function $w=f_{2}$ satisfies either (21) or (22).

First of all we assume that $(21)$ is not irreducible. Then there exists a singlevalued meromorphic function $w=w_{1}(X)$ satisfying (21). It is easy to verify that there exists no common zero of $4 y_{0}^{3} X^{12}+27 A^{2}$ and $4 x_{0}^{2} X^{6}-3(4 d-3) B^{2}$. We assume that there is a finite pole of $w=w_{1}(X)$, say $X_{0}$, which is of order $p$, then $X_{0}$ is a zero of $4 y_{0}^{3} X^{12}+27 A^{2}$. By (21) we have $p=1 / 2$, which is absurd. Hence $w=w_{1}(X)$ has no pole on $C$. Next let us put $X=1 / t$, then we have

$$
\begin{gathered}
2\left(4 y_{0}^{3}+27 A^{2} t^{12}\right) w^{3}-\frac{3}{2} y_{0}\left(4 x_{0}^{2}-3(4 d-3) B^{2} t^{6}\right) t^{2} w \\
+\left(-2 x_{0}^{3}+9 d B^{2} x_{0} t^{6}+27 A K t^{9}\right) t^{3}=0
\end{gathered}
$$

from (21). Therefore $w=w_{1}(X)$ has a simple zero over $X=\infty$. Therefore we have $w=w_{1}(X) \equiv 0$ by Liouville's theorem. This is a contradiction. Hence the equation (21) is irreducible. So we can consider the 3-valued algebraic function defined by (21). The function $w=w(X)$ has 12 poles on $\{X \mid$ $\left.4 y_{0}^{3} X^{12}+27 A^{2}=0\right\}$, all of which are algebraic branch points of order 1 . Therefore the compact Riemann surface, defined by $w=w(X)$, is of genus $g \geqq 4$. By lemma 1, there exist no pair of meromorphic functions $X=e^{J}$ and $w=f_{2}$ satisfying the equation (21). This is absurd.

Next let us consider (22). And let us assume that (22) is not irreducible. Then there exists a single-valued meromorphic function $w=w_{2}(X)$ satisfying (22). It is easy to verify that there exists no common zero of $4 y_{0}^{3} X^{12}+27 A^{2}$ and $3(4 d-3) B^{2} X^{6}-4 x_{0}^{2}$. We assume that there is a finite non-zero pole of $w=$ $w_{2}(X)$, say $X_{0}$, which is of order $p$, then $X_{0}$ is a zero of $4 y_{0}^{3} X^{12}+27 A^{2}$. And by (22) we have $p=1 / 2$, which is absurd. Hence $w=w_{2}(X)$ has only one pole at $X=0$, which is of order 3. Putting $X=1 / t$, we have

$$
\begin{gathered}
2\left(4 y_{0}^{3}+27 A^{2} t^{12}\right) w^{3}+\frac{3}{2} y_{0}\left(3(4 d-3) B^{2}-4 x_{0}^{2} t^{6}\right) t^{8} w \\
+\left(9 d B^{2} x_{0}+27 A K t^{3}-2 x_{0}^{3} t^{6}\right) t^{15}=0
\end{gathered}
$$

from (22). Therefore $w=w_{2}(X)$ has a zero of order at least 4 at $X=\infty$. This is a contradiction. Hence the equation (22) is irreducible. So we can consider the 3-valued algebraic function $w=w(X)$ defined by (22). The function $w=$ $w(X)$ has 12 branch points of order 1 on $\left\{X \mid 4 y_{0}^{3} X^{12}+27 A^{2}=0\right\}$, therefore the compact Riemann surface, defined by $w=w(X)$, is of genus $g \geqq 4$. By lemma 1 , there exist no pair of meromorphic functions $X=e^{J}$ and $w=f_{2}$ satisfying the equation (22). This is absurd. Therefore there exists no entire function $f$ on $\boldsymbol{R}_{A^{-}}$-(i), which defines the surface $\boldsymbol{X}$-(i).

By the similar way of above, we can verify that there exists no single-valued meromorphic function $w=f_{2}$ satisfying each of the equations (23), (24), (25) and (26). Therefore there exists no entire function $f$ on $\boldsymbol{R}_{A}$-(i), which does not take 5 finite values. Hence $\boldsymbol{R}_{A}$-(i) is of Picard constant 5 . 
The similar way of above remains valid in the case of $\boldsymbol{R}_{A}$-(ii). Q.E.D

Acknowledgement. The author wishes to thank the referee for many valuable comments and suggestions.

\section{REFERENCES}

[1] H. AogaI, Picard constant of a finitely sheeted coverıng surface, Kōdaı Math. Sem. Rep., 25 (1973), 219-224.

[2] W K. Hayman, Meromorphic Functions, London, Clarendon Press, 1964.

[ 3 ] G. HiRomi AND K. NiINO, On a characterization of regularly branched three-sheeted covering Riemann surfaces, Kōdaı Math. Sem. Rep., 17 (1965), 250-260.

[4] R. Nevanlinna, Eindeutıge analytısche Funktionen. Sprınger-Verlag, Berlin, 1st ed. 1936, 2nd ed. 1953. Analytic Functions (English version). Sprınger-Verlag, Berlin, 1970.

[5] M. Ozawa, On complex analytıc mappıngs. Kōdaı Math. Sem. Rep., 17 (1965), 93-102.

[6] M. Ozawa, On ultrahyperelliptıc surfaces. Kōdaı Math. Sem. Rep., 17 (1965), 103-108.

[7] M. Ozawa AND K. SAwada, Three-sheeted algebrold surfaces whose Picard constants are five. Koda1 Math. J., 17 (1994), 101-124.

[ 8 ] E. PICARD, Démonstration d'un théorème général des fonctions uniformes liées par une relation algébrique. Acta Math., 11 (1887), 1-12.

[9] K. Sawada AND K. Tohge, A remark on three-sheeted algebrold surfaces whose Picard constants are five. Kodal Math. J., 18 (1995), 142-155.

[10] H. L. SelberG, Algebroide Funktionen und Umkehrfunktionen Abelscher Integrale. Avh. Norske Vid. Akad. Oslo, 8 (1934), 1-72.

DEPARTMENT OF MATHEMATICS

Tokyo Metropolitan College of Technology

SHINAGAWA, TOKYO, JAPAN

e-mail: sawada@tokyo-tmct.ac.jp 